Revue d'histoire de l'enfance « irrégulière »

Le Temps de l'histoire

$20 \mid 2018$

Sexualités juvéniles

\title{
L'arrivée des éducateurs dans les institutions de rééducation pour filles : une mise en scène de l'hétéronormativité
}

The Arrival of Male Workers in Reform Schools for Girls: Acting of

Heteronormativity

Pauline Tournier

(2) OpenEdition

Journals

Édition électronique

URL : http://journals.openedition.org/rhei/4601

DOI : $10.4000 /$ rhei. 4601

ISSN : $1777-540 \mathrm{X}$

Éditeur

Presses universitaires de Rennes

Édition imprimée

Date de publication : 15 novembre 2018

Pagination : 151-177

ISBN : 978-2-7535-7571-4

ISSN : $1287-2431$

\section{Référence électronique}

Pauline Tournier, «L'arrivée des éducateurs dans les institutions de rééducation pour filles : une mise en scène de l'hétéronormativité », Revue d'histoire de l'enfance « irrégulière » [En ligne], 20 | 2018, mis en ligne le 15 novembre 2020, consulté le 10 décembre 2020. URL : http://journals.openedition.org/rhei/ 4601 ; DOI : https://doi.org/10.4000/rhei.4601 


\section{L’arrivée des éducateurs dans les institutions de rééducation pour filles: une mise en scène de l'hétéronormativité}

Jusqu'à la fin des années soixante, les institutions de rééducation pour filles sont encadrées uniquement par des éducatrices, religieuses ou laïques. Celles-ci se doivent d'être célibataires et entièrement dévouées à leur mission éducative. L'arrivée des premiers éducateurs, qui apparaît dans un contexte marqué par d'importants bouleversements au sein de la société et notamment dans la jeunesse, entraîne une redéfinition de ce modèle professionnel. Les liens du mariage et l'affirmation d'une vie sexuelle épanouie, sous le contrôle de l'institution, sont désormais jugés préférables. En outre, cette présence d'éducateurs a des conséquences sur les méthodes pédagogiques. En effet, la mixité apparaît comme un nouveau moyen de corriger les inadaptations de ces jeunes filles; inadaptations liées à leur genre ou à leurs comportements sexuels. Cet article s'attache à montrer comment la présence des éducateurs va permettre, en s'appuyant sur des arguments psycho-pédagogiques, à la fois de réaffirmer la différence des sexes et de maintenir la sexualité dans un cadre hétéronormatif.

Until the end of the sixties, Reform Schools for girls were supervised by female youth workers only, nuns or lay professionals. They had to be single and fully dedicated to their educational mission. The arrival of the first male youth workers, which appears in a context of major unrest in society and especially in youth, led to a redefinition of this professional model. The bonds of marriage and the affirmation of a fulfilling sexual life, under the institutional control, were considered preferable. Furthermore, this presence of male youth workers would also impact pedagogy. Indeed, the co-education would be used as a new educational tool to correct the maladjustment of girls; maladjustment linked to their gender and their sexuality. This article aims to show how this presence of male youth workers will allow, using psycho-pedagogical point of view both to reaffirm the differences between sexes, and to maintain sexuality in a heternormative framework.

Mots-clés : mixité, genre, sexualité, rééducation, familles.

Keywords: co-education, gender, sexuality, reeducation, families.

\author{
Pauline Tournier \\ Titulaire d'un master 2 \\ en études de genre, \\ université Paris 8. \\ Chargée de mission \\ pour le portail numé- \\ rique « Enfants en \\ justice » et confé- \\ rencière au Centre \\ d'exposition historique \\ de Savigny (ENPJJ)
}


1. Voir notamment Rogers Rebecca (dir.). La mixité dans l'éducation : enjeux passés et présents, Lyon, ENS, 2004

2. Schweitzer Sylvie, Les femmes ont toujours travaillé. Une histoire du travail des femmes aux XIX et $x x^{e}$ siècles, Paris, Odile Jacob, 2002.

3. Les Bons Pasteurs sont des congrégations religieuses nées au milieu du xIx siècle et qui ont eu jusqu'aux années 1960 le monopole de la rééducation des filles.

4. Gardet Mathias, Peyre Vincent, Tetard Françoise (dir.), Elles ont épousé l'éducation spécialisée. Éducatrices et femmes d'éducateurs il y a cinquante ans, Conservatoire National des Archives et de l'Histoire de l'Éducation Spécialisée, L'Harmattan, 1999.

\section{Ibid.}

6. Voir Perrot Michelle, Les femmes ou les silences de l'Histoire, Paris, Flammarion, 1998.
T 'histoire de la justice des mineuree's a longtemps été une histoire de la non-mixité. Tout au long du XIX ${ }^{\mathrm{e}}$ siècle et jusqu'au milieu du $\mathrm{Xx}^{\mathrm{e}}$, à l'instar de l'institution scolaire ${ }^{1}$ ou du monde du travail ${ }^{2}$, la séparation des sexes, fondée sur l'idée d'une hiérarchisation naturelle entre hommes et femmes, représente la norme. Que ce soit dans les colonies pénitentiaires, les prisons pour mineurs, ou les maisons de correction, ce sont donc les surveillants, moniteurs et autres encadrants masculins qui prennent en charge les garçons, quand les filles, elles, sont confiées aux religieuses des congrégations comme le Bon Pasteur ${ }^{3}$. L'ordonnance du 2 février 1945 qui créé l'Éducation surveillée (ES) - ancêtre de la Protection judiciaire de la jeunesse (PJJ) - en tant qu'entité autonome de l'Administration pénitentiaire, va entraîner de nombreux changements, comme l'apparition et la professionnalisation d'un nouveau personnel éducatif et la naissance de nouvelles institutions. Mais elle ne remet pas en cause cette ségrégation sexuée. Il faut attendre les années soixante, au moment où la mixité s'impose, et comme élément de langage, et comme principe de réalité dans les établissements scolaires de l'Éducation nationale, pour voir apparaître les premières expériences de mixité au sein de l'Éducation surveillée, et ce, d'abord du côté des professionnel·le·s, avec la présence d'éducatrices dans les établissements masculins, puis l'arrivée des éducateurs dans les équipes féminines. Les différentes lois sur l'égalité professionnelle accélèrent le processus à partir des années soixante-dix et aujourd'hui, cette présence de professionnellle·s des deux sexes au sein de la PJJ apparaît normale, évidente, acquise, à l'instar d'autres secteurs professionnels.

Comment ce passage d'un univers unisexué à la généralisation des équipes mixtes a-t-il été possible et comment l'analyser au regard des enjeux éducatifs, et du contexte de l'époque?

Très peu de recherches concernant la justice des mineur·ess sont postérieures aux années soixante et il n'existe quasiment rien pour l'heure sur cette arrivée de la mixité dans les établissements, mis à part «Elles ont épousé l'Éducation surveillée ${ }^{4}$ », qui donne la parole à d'anciennes éducatrices ayant travaillé dans des institutions pour garçons. "Le secteur de la Rééducation, tel qu'il est formalisé et développé avant, pendant et après la seconde guerre, est pleins de récits de pionniers qui sont d'abord des récits d'hommes ${ }^{5}$ ". Ceci explique sans doute pourquoi dans ce champ de recherche aussi, les femmes ont mis du temps à sortir du silence de l'histoire ${ }^{6}$ et combien les travaux menés par Anne 
Thomazeau sur les éducatrices ${ }^{7}$ ou par Véronique Blanchard sur les « mauvaises filles ${ }^{8}$ " sont somme toute encore assez récents. C'est donc dans le prolongement de cette histoire que j'ai souhaité interroger l'impact que pouvait représenter l'entrée des éducateurs dans les institutions féminines, à la fin des années soixante.

De nombreuses recherches en sociologie ou en histoire ont étudié l'organisation des rapports sociaux de sexe pour mettre en évidence l'existence d'une division sexuelle du travail mais aussi la façon dont le genre impacte différemment les pratiques professionnelles et les rapports de pouvoir, dès lors qu'il s'agisse de femmes dans des métiers majoritairement masculins, ou d'hommes investissant des métiers pensés comme féminins ${ }^{9}$. L'histoire de l'éducation a surtout abordé la mixité du côté du public et au sein de l'Éducation nationale, mais les travaux notamment de Rebecca Rogers ont permis de montrer combien ce fait social était toujours historiquement situé et affaire de politique pédagogique ${ }^{10}$. C'est pourquoi, il est nécessaire de considérer cette arrivée des éducateurs, non seulement au regard des normes de genre du côté des professionnellle·s qu'elle redessine, mais aussi à l'aune des enjeux spécifiques de la rééducation des filles et du contexte, aussi bien général, que particulier à l'ES.

Depuis l'ordonnance de 1945, les institutions de rééducation de l'Éducation surveillée sont enjointes à privilégier l'éducatif sur le répressif. Chargées d'accueillir des mineur·e's placée's par le juge pour enfants pour des faits de délinquance et/ou à protéger, elles ont pour mission de remettre sur « le droit chemin » ces enfants perdus, en engageant des professionnel-le·s : éducateurs, mais aussi psychologues, médecins, instructeurs, etc. Dans les internats professionnels de l'Éducation surveillée (IPES) qui accueillent des adolescent·e-s de 13 à 21 ans, la formation professionnelle occupe une place importante. Au cours des Trente Glorieuses, la tendance est à la réinsertion des jeunes. Il faut ouvrir les institutions sur l'extérieur. Ces jeunes, ce sont aussi les nouveaux éducateurs et les nouvelles éducatrices qui entrent en formation et qui bousculent l'Éducation surveillée. Cette ouverture est également vraie du côté des filles, mais les évolutions sont nettement plus lentes. Jusqu'à la fin des années 1960, leur prise en charge est assurée essentiellement par des congrégations religieuses, l'État considérant que ce sont là, qu'elles pourront trouver leur salut. Délinquantes, déviantes, ces jeunes filles sont perçues comme doublement coupables : pour avoir enfreint la loi mais aussi moralement, pour avoir transgressé le rôle social
7. Thomazeau Anne, « Entre éducation et enfermement : le rôle de l'éducatrice en internat de rééducation pour filles, de la Libération au début des années 1960 ", Revue d'histoire de l'enfance "irrégulière ", $\mathrm{n}^{\circ} 7,2005$, mis en ligne le 6 juin 2007.

8. Blanchard Véronique, "Mauvaises filles": portaits de la déviance féminine juvénile (1945-1958), thèse de doctorat en histoire, sous la dir. de Frédéric Chauvaud, Poitiers, 2016.

9. Voir KeRgoat Danièle, VILBROd Alain, GuichardCLAUDIC Yvonne (sous la dir.), L'inversion du genre. Quand les métiers masculins se conjuguent au féminin... et réciproquement, PUR, 2008.

10. Rogers, La mixité dans l'éducation..., op. cit. 
de femme qui leur est assigné. Pour les corriger, il convient de les isoler dans des institutions fermées et de leur éviter toute fréquentation avec l'autre sexe. Les éducatrices, religieuses et laïques, ont pour mission de leur apprendre à devenir une bonne épouse et une bonne mère au sortir de l'institution. La méthode : être un modèle d'identification féminine, tout entière dévouée à son travail, à la moralité et aux mœurs irréprochables.

L'arrivée des éducateurs dans ces institutions à partir de la fin des années 1960 représente donc un véritable retournement de ces principes éducatifs. Pourtant, alors que l'entrée des femmes dans les internats pour garçons provoque certaines résistances, cette mixité ne suscite aucun désaccord ni véritable débat. Au contraire, les rares discussions qui apparaissent dans les archives la qualifient de progrès et l'associent à un processus de normalisation des internats. Alors que l'Éducation surveillée est en proie à de nombreux changements (accroissement des effectifs, émergence d'une nouvelle génération de professionnel-le·s, remise en cause des méthodes autoritaires et des grosses structures, etc.), que les mouvements féministes et les revendications d'une plus grande libération sexuelle battent leur plein dans la société, comment comprendre cette réaction positive à l'entrée des hommes et que vient signifier la mixité pour les acteurs-trice-s de l'époque? Dans des institutions fermées où toutes les éducatrices sont nécessairement célibataires, quel encadrement des relations sexuelles et quelles modifications des normes observe-t-on pour les professionnel-le·s? La rééducation des jeunes filles se centrant principalement sur le traitement de leurs déviances sexuelles, il est également nécessaire d'interroger la mixité dans le cadre de ces enjeux éducatifs. En outre, inscrite dans des rapports de pouvoir entre institution judiciaire et milieu populaire, les normes sexuelles et le système de genre qui en découle s'articulent nécessairement avec la question sociale et l'ordre familial. C'est au prisme de ces communications entre dedans et dehors, de ces points de tensions entre répression et transformation des comportements du sujet féminin, et de ces passages entre privé et professionnel, que la question de l'articulation entre mixité et sexualité juvénile sera analysée.

Cet article, issu de mon mémoire de recherche, s'appuie sur plusieurs sources, et en premier lieu, les rapports annuels de deux institutions publiques emblématiques pour la période, à savoir l'IPES de Brécourt et celui de Bourges. Le premier est créé en 1947, et constitue pendant longtemps la seule institution publique féminine. Sa directrice Dominique Riehl va, dès le début, défendre un 
modèle de rééducation spécifique aux filles, sur lequel nous aurons l'occasion de revenir. Le deuxième est racheté par l'État en 1967 après avoir longtemps appartenu aux Bons Pasteurs. Il s'agit donc de deux institutions aux histoires bien différentes. Ont également été mobilisées les archives du Centre de Vaucresson, et plus particulièrement les publications scientifiques de la sociologue Andrée Algan, ainsi que les comptes rendus des journées d'étude portant sur la rééducation des filles et la question de la mixité, et les mémoires rédigés par les élèves en formation. Enfin, dans le cadre de mon mémoire, trois entretiens ont été menés avec d'anciennes éducatrices, toutes passées par Brécourt ou Bourges au cours des années soixante et soixante-dix. Si seul un court extrait est repris ici, il est important pour moi de préciser que ces témoignages ont occupé une place non marginale dans mon analyse. En effet, peu de traces écrites subsistent de ces professionnelles, les rapports annuels ayant été rédigés par la direction et les écrits des professionnels étant majoritairement rédigés par des hommes.

\section{LA MIXITÉ DES ÉQUIPES : DU MARIAGE À L'INSTITUTION}

\section{À L'INSTITUTION DU MARIAGE}

\section{Du célibat à la vie de couple : une émancipation hors de l'institution}

Jusqu'au milieu des années soixante, le célibat constitue l'horizon indépassable des éducatrices, qu'elles soient religieuses ou laïques. Cette situation, commune à d'autres professions comme les infirmières, les assistantes sociales ou encore les institutrices, n'est pas nouvelle ${ }^{11}$, mais elle est renforcée par le contexte de l'après-guerre. Les politiques natalistes et familialistes cherchent en effet à maintenir les mères au foyer, renvoyant l'engagement dans la vie professionnelle à une véritable vocation, peu compatible avec le fait de fonder une famille ${ }^{12}$. Dans ces lieux d'enfermement que constituent les internats de l'ES, les éducatrices dorment la plupart du temps dans l'enceinte de l'établissement, et leur emploi du temps, rythmé par la vie de l'institution, ne leur laisse aucun répit pour sortir ou profiter de leur temps libre et avoir une vie privée. La surveillance et l'isolement des jeunes filles pèsent aussi sur les éducatrices ${ }^{13}$.

Mais le célibat n'est pas seulement un état de fait. À Brécourt, il est également théorisé comme un véritable " moyen de rééducation ${ }^{14}$ ». Contrairement aux IPES pour garçons qui peuvent volontiers accueillir des "femmes mariées " voire des « couples d'éducateurs-éducatrices », pour Dominique Riehl, la direc-
11. Voir à ce sujet: GuILPAIN Geneviève, Les célibataires, des femmes singulières. Le célibat en France

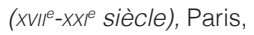
L'Harmattan, 2012.

12. En 1954 , seules $24,7 \%$ des mères ayant deux enfants travaillent, selon Courson Jean-Pierre et SABoulin Michel, « Ménages et familles : vers de nouveaux modes de vie? ", Économie et statistiques, $n^{\circ} 175,1985$, p. 13. Voir aussi l'article de LEFAUCHEUR Nadine, 1992, "Maternité, famille, État », in DuBY Georges, Perrot Michelle (sous la dir.), Histoire des femmes en Occident, tome 5 : Le $x x^{e}$ siècle, Paris, Plon, 1991, p. 411-430.

13. Voir Thomazeau, «Entre éducation et enfermement... », op. cit.

14. RIEHL Dominique, "L'éducatrice d'IPES ", compte rendu d'une conférence de la directrice de l'IPES de Brécourt, session de formation des éducateurs, 1947, Archives de Savigny-sur-Orge, sans cote, p. 10. 
15. RIEHL «L'éducatrice d'IPES... ", op. cit.

16. Ibid.

17. BLANChard Véronique, Revenin Régis, "Justice des mineurs, travail social et sexualité juvénile dans le Paris des années 1950 : une prise en charge genrée ", Les Cahiers de Framespa [en ligne], 7, 2011, mis en ligne le 6 avril 2011.

18. RIEHL, ibid.

19. Voir les Assises nationales de l'Éducation surveillée qui se tiennent à Vaucresson du 24 au 28 juin

1968.

20. Lagrave Rose-Marie, « Une émancipation sous tutelle. Éducation et travail des femmes au xxe siècle ", in Duby Georges, Perrot Michelle (sous la dir.), Histoire des femmes... op. cit. trice, " une femme mariée ne peut pas remplir dans un IPES de filles le rôle d'éducatrice ou d'éducatrice adjointe [...] L'éducatrice ne peut être à la fois mère de famille dans son propre foyer et mère de famille des 7 adolescentes avec qui elle occupe un appartement et avec lesquelles elle doit former un véritable foyer familial modèle ${ }^{15}$ ". Les éducatrices se doivent d'être dévouées à l'institution, comme une épouse l'est à son foyer. Outre le rôle maternel, Dominique Riehl attend de celles-ci qu'elles incarnent un modèle de féminité pour les jeunes filles. C'est le principe de la pédagogie par l'imitation. Les éducatrices doivent adopter un comportement irréprochable, refléter l'idéal de vertu féminine qui permettra de remettre ces « mauvaises filles » dans le droit chemin. Selon Dominique Riehl, "le malheur de nos pupilles a son origine dans les relations sexuelles ${ }^{16}{ }$. Cette affirmation reflète les motifs de placement des jeunes filles dans les années cinquante. L'étude des dossiers judiciaires et l'analyse des décisions prises à leur encontre montrent combien la question de la sexualité est déterminante, puisqu'elle est majoritairement à l'origine de la prise en charge ${ }^{17}$. On comprend mieux dès lors pourquoi le redressement du corps féminin, la correction des déviances de ces jeunes filles supposent la construction d'un espace non-mixte d'où la sexualité doit être absente. L'abstinence aussi doit être incarnée par les éducatrices : "J'ai dit tout à l'heure que nous devions prouver à nos filles qu'il y a moyen de vivre sans relations sexuelles ${ }^{18}$. "

Or, en 1965, le rapport annuel de Brécourt fait apparaitre l'embauche d'une première éducatrice mariée. Puis, à partir de 1968, la direction mentionne la nécessité de recruter des ménages ou des éducateurs mariés.

Plusieurs facteurs permettent d'expliquer cette décision. À partir de la fin des années soixante, les internats sont de plus en plus décriés au sein de l'ES ${ }^{19}$. L'isolement et la lourdeur qui les caractérisent s'adaptent mal à la volonté d'ouvrir de plus petites structures, davantage ancrées dans la société. De leur côté, les directions peinent à renouveler leurs équipes. Le profil des éducatrices change. Les femmes, qui sont plus nombreuses à accéder aux études secondaires, et aux formations professionnelles ${ }^{20}$, revendiquent de pouvoir concilier vie familiale et exercice de leur métier. Elles restent peu dans ces grands internats. Les équipes sont donc pour beaucoup composées de jeunes professionnelles qui n'ont guère d'écart d'âge avec les jeunes filles placées. Les contradictions entre la représentation attendue d'une mère et épouse parfaite et le profil de ces jeunes éducatrices supposées chastes et célibataires, sans aucune expérience d'une vie 
de famille apparaissent plus nettement : "Comment peut-on sérieusement à 20 ans, à 25 ans, apporter à de jeunes filles de 14 à 18 ans l'image sécurisante d'une mère ${ }^{21}$ ? » Ces contraintes de genre et de sexualité qui pèsent sur les jeunes éducatrices fonctionnent d'autant moins quand, à partir de la fin des années soixante, la jeunesse commence à occuper une place importante dans la société, se fait entendre, et notamment pour revendiquer plus de liberté. Les événements de Mai 68 n'épargnent pas l'Éducation surveillée. Les Assises nationales de l'ES qui se tiennent entre le 24 et le 28 juin font suite à une série de grèves et d'occupations. Les éducatrices en formation à Brice-la-Forêt (depuis 1965, les filles et les garçons sont reçus dans deux écoles séparées) n’hésitent pas à descendre au Centre de formation de Savigny-sur-Orge où sont installés les éducateurs pour participer à l'occupation ${ }^{22}$. L'année suivante, les deux promotions sont réunies.

Cette arrivée des éducateurs peut apparaître dès lors comme une mesure contrainte par les événements. Elle sera vite défendue par les directions avec des arguments plus éducatifs. À Bourges, le projet insiste sur l'importance de moderniser son institution. La mixité viendrait refléter la vie en société telle qu'elle existe de l'autre côté des murs :

L'équipe des responsables s'était accordée au départ sur deux perspectives 《fondamentales : - le "désenfermement", l’ouverture sur l'extérieur [...] ; au lieu de recréer un monde clos défini à partir d'un code spécifique, nous étions résolus à introduire à l'intérieur de l'établissement les conditions mêmes de la réalité et de la vie normale; ceci eut par exemple pour première conséquence la mixité du personnel amorcée dès l'ouverture de l'établissement ${ }^{23}$. »

L'embauche d'éducateurs permettrait en outre de stabiliser des équipes, qui sont, d'après la direction, fragilisées par l'immaturité et l'instabilité des jeunes professionnelles : "La présence masculine amène une certaine émulation et peut constituer un élément rassurant, pour les éducatrices très jeunes dans le métier ${ }^{24}$. " Mais les éducateurs qui arrivent ne sont pas choisis au hasard. Que ce soit à Bourges ou à Brécourt, ils sont mariés à des éducatrices déjà en poste ${ }^{25}$.

La mixité des équipes signe donc la fin du célibat obligatoire et la possibilité pour les éducatrices d'avoir une vie sexuelle. En outre, le développement de logements à l'extérieur de l'institution, et même en plein cœur de la ville comme à Bourges, pour accueillir ces nouveaux ménages, permet aux éducatrices d'avoir un espace à elles, une vie privée hors des murs.

\author{
21. «Bulletin du Syndicat \\ National des Personnels \\ de l'Éducation Surveillée ", \\ $n^{\circ}$ 46, décembre 1964, \\ archives de Savigny-sur- \\ Ogre, sans cote. \\ 22. Voir notamment le \\ témoignage de Claire \\ Dumas sur Criminocorpus \\ [https://criminocorpus.org/ \\ media/filer_public/d4/fa/ \\ d4faf8ec-4e9f-4849-823f- \\ 3f5c654f3916/mbasdevant \\ cdumas.pdf]. \\ 23. Pretot Renée, \\ "Hypothèse de travail : le \\ complexe éducatif ", in Les \\ Annales de Vaucresson, \\ $n^{\circ} 11,1973$, p. 12. \\ 24. Rapport annuel de \\ Bourges, "Section IV. \\ Internat, remarques \\ générales sur l'organisa- \\ tion pédagogique ", p. 5 , \\ 1971, Archives natio- \\ nales de Pierrefitte, cote \\ 19910298/81. \\ 25. En 1972 par exemple, \\ I'IPES de Brécourt compte \\ 5 éducateurs, tous mariés. \\ Voir le « Rapport annuel \\ de l'IPES de Brécourt, \\ Conclusion du directeur ", \\ rédigé par Madame Beaulu, \\ 1972, Archives Nationales \\ de Pierrefitte, cote \\ 19910298/65. Et à Bourges, \\ c'est dès le rachat par l'État \\ en 1967 qu'apparaissent \\ deux éducateurs, eux aussi \\ mariés.
}


26. «Sexe : sous la révolution, les normes », Mouvements, vol. 20, n², 2002, p. 9-14

27. Voir aussi le témoignage de Claire Dumas sur Criminocorpus :

[https://criminocorpus.org/ media/filer_public/d4/fa/ d4faf8ec-4e9f-4849-823f3f5c654f3916/mbasdevant cdumas.pdf].

28. Voir à ce sujet : Artieres Philippe, ZANCARINI-FOURNEL Michelle (dir.), 1968, une histoire collective (19621981), Paris, La Découverte, 2008.

29. Thomazeau, « Entre éducation et enfermement... », op. cit.

30. Rapport envoyé au ministère de la Justice, par le Centre de formation et de recherche de l'Éducation surveillée de Vaucresson, Journées des 6 et 7 mai 1971. p. 7, Archives nationales de Pierrefitte, cote 20060575/002.
Pour autant, cette nouvelle conjugalité rendue possible est-elle synonyme de libération sexuelle? Dans quelle mesure, cet assouplissement des contraintes sexuelles représente-t-il une réelle émancipation hors de l'institution?

\section{Une sexualité sous contrôle}

"La Révolution sexuelle a-t-elle eu lieu ${ }^{26}$ ? " Ainsi, s'interrogent nombre de chercheurs travaillant sur les " années 68 ». Il est difficile de se distancer du vocabulaire emprunté par les acteurs et actrices de l'époque pour commenter cette période, tant la vision d'une transformation radicale, d'un basculement entre deux époques, a marqué les consciences et produit une forme de récit mythique. Ainsi, les éducatrices en formation à partir de 1967 que j'ai pu interroger ont invoqué "les événements de 68 » comme un fait marquant, ayant eu une influence sur leur propre parcours, ainsi que dans les institutions féminines ${ }^{27}$. Les mouvements féministes qu'elles ont rencontrés, parfois en tant que militantes, le développement des cours d'éducation sexuelle, la « révolution contraceptive " ou encore la libération de la parole ont représenté autant de symboles d'une émancipation sexuelle, non seulement des jeunes filles, mais également pour elles-mêmes. Il convient néanmoins de replacer cette époque dans un contexte plus large. Mai 68 serait moins un événement "magique", qu'une exacerbation voire une accélération des évolutions déjà commencées au cours des années soixante ${ }^{28}$. Ensuite, en questionnant davantage les constances, les résistances et les nouvelles formes de contrôle et de normes qui se maintiennent et apparaissent.

À l'inverse des éducatrices, «le célibat des éducateurs n'est pas théorisé comme un moyen de rééducation. Au contraire, le soupçon pèse, passé un certain âge, sur l'homme célibataire qui souhaite travailler auprès d'enfants et de jeunes gen ${ }^{29}{ }^{\prime}$. Ce propos vaut aussi pour ceux qui choisissent de travailler dans les institutions féminines. Les réactions des éducateurs de garçons vis-àvis des volontaires qui vont travailler auprès de jeunes filles sont teintées de méfiance : «cette orientation leur apparaît suspecte, tout au moins dans les premières années de vie professionnelle (motivations sexuelles plus ou moins camouflées ${ }^{30}$ ) ». En outre, l'écart d'âge est parfois mince; et ces jeunes filles continuent toujours d'être perçues comme déviantes sexuellement. C'est pourquoi les participant.e.s aux journées de Vaucresson considèrent que l'éducateur, davantage que ses collègues féminines, se doit d'être vigilant, dans les relations 
qu'il instaure avec ces adolescentes : "il ne faut pas négliger le jeu subtil de séduction qui inconsciemment colore certains de ces échanges ${ }^{31}$ ". Certaines institutions adoptent alors des mesures d'évitement. Le rapport publié en 1977 par Andrée Algan en cite quelques-unes : les éducateurs ne sont chargés que des activités de la journée, " celui-ci devrait éviter les situations ambiguës (les filles cherchent souvent à l'y entraîner), et à faire en sorte de toujours rester maître de ses relations avec l'adolescente ${ }^{32}$ ". Une certaine division du travail se met donc en place afin d'éviter aux éducateurs d'avoir des contacts trop intimes avec les jeunes filles, (faisant ainsi reposer les tâches du care et du soin uniquement sur les femmes).

Cette vigilance reflète la " panique morale » qui s'empare de ces institutions. Étant donné le profil de ces jeunes filles, leur proximité d'âge avec les éducateurs et l'évolution des conduites sexuelles, il est tout à craindre de relations entre professionnels et adolescentes. D'autant que certains éducateurs seraient prêts à remettre en question cet interdit. À la fin des années soixante-dix, à la faveur d'une libération de la parole qui s'autorise à questionner le droit à la sexualité des enfants, et notamment la notion de majorité sexuelle ${ }^{33}$, transparaissent en effet dans les rapports d'Andrée Algan des interrogations assez nouvelles :

Certains se demandent si le refus de l'éducateur de passer à l'acte avec un 《 jeune de l'autre sexe ne peut pas parfois avoir des conséquences aussi traumatisantes pour le jeune qu'un passage à l'acte lorsque la relation a été très loin. Le refus est difficile à accepter pour une fille, par exemple ${ }^{34}$. "

Ces propos apparaissent néanmoins de manière très marginale dans les écrits que j'ai pu consulter, ce qui est confirmé par la chercheuse de Vaucresson :

Quelques éducateurs remettent en question l'interdit qui pèse sur les rela《tions sexuelles entre éducateur et adolescente; mais il semble que dans la quasi-totalité des cas, il ne s'agisse que d'une transgression théorique de la règle, sans réel passage à l'acte ${ }^{35}$."

Ces tentations relationnelles restent perçues comme une menace pour l'institution. Embaucher des éducateurs de préférence mariés, stables sexuellement, suffisamment matures pour ne pas être tentés par des relations avec les jeunes

\author{
31. "Journées d'études \\ sur les structures d'action \\ éducative destinées \\ aux filles inadaptées \\ Vaucresson- 20-22 mars \\ 1972 », p. 8, Archives natio- \\ nales de Pierrefitte, cote \\ 20060575/002.
}

32. Algan Andrée, La mixité du groupe dans les institutions accueillant des jeunes inadaptés. Rapport d'enquête, Éd. Vaucresson, Centre de formation et de recherche de l'Éducation surveillée, avril 1977, p. 51, CNAM, cote MO 8044.

33. Voir Berard Jean, « De la libération des enfants à la violence des pédophiles. La sexualité des mineurs dans les discours politiques des années 1970 ", Genre, sexualité \& société, n० 11 , printemps 2014.

34. Algan Andrée,

"Compte-rendu de la session sur la mixité du groupe dans les institutions accueillant des jeunes inadaptés. Vaucresson, du 16-20/1/1978 ", mars 1978, p. 7, Archives de Savignysur-Orge, sans cote.

35. Ibid. 
36. Algan, La mixité du groupe..., op. cit.

37. «Colloque de 1968 sur la formation de l'éducateur à Vaucresson en février 1968, organisé par le comité d'entente des écoles et centres de formation d'éducation spécialisée : session sur sélection des candidats éducateurs en 1968 , rapporté par $\mathrm{M}^{\text {le }}$ Bauer ", in Sauvegarde de l'enfance, 12 , n553, 1968, CEDIASMusée social.

38. Docteur LE MOAL, "Conférence Mixité et Coéducation », Centre catholique d'éducation familiale (France), 1968, Bibliothèque nationale de France, cote O-R-62300 (1967-1968,5) filles, et au clair avec leurs désirs (hétéro)sexuels est dès lors considéré comme une assurance morale.

Bien plus, cette sexualité est gage de qualités professionnelles. En effet, selon Andrée Algan, la mixité des équipes dépend de «la manière dont l'éducateur vit son propre sexe et son état (célibat ou mariage plus ou moins réussi) ${ }^{36}$ ". Il est important que les éducateurs aient une vie sexuelle épanouie, ou en tout cas d'avoir réglé leurs " problèmes sexuels ", dont celui de l'« identité sexuelle ». L'incitation de la directrice à Bourges concernant la vie sociale des éducatrices peut être également comprise dans ce sens-là. Être une bonne éducatrice passerait désormais par le fait d'avoir une vie privée satisfaisante. La sexualité fait donc l'objet d'un investissement positif et c'est, à l'inverse, son absence qui peut être source d'inquiétude.

Les années d'après-guerre se distinguent par un renforcement des mesures contre l'homosexualité, et l'émergence de discours médicaux, accompagnant l'idée qu'il s'agit d'une maladie mentale. L'inscription de l'homosexualité au registre de l'OMS en 1968 vient alors entériner cette ligne répressive. Ainsi, à l'Éducation surveillée, il est spécifié dans les critères de sélection des candidatséducateurs de 1968 : «Dans cette perspective, qui pouvons-nous éliminer sans regrets? - les psychopathes, sans contestation possible - les homosexuels, bien entendu ${ }^{37}$. " L'attirance pour les hommes est à cette époque, incompatible avec la prise en charge éducative de jeunes. Dans cette optique, les liens conjugaux représenteraient ici encore un gage de moralité. Et si les années soixante-dix voient émerger des mouvements homosexuels et lesbiens importants dans la société, remettant en cause le système hétérosexiste et " hétéroflic ", les rares écrits laissés par les élèves-éducateurs-trices en formation qui défendent le droit à l'homosexualité le revendiquent pour les garçons accueillis, jamais sous l'angle d'un possible pour les éducateurs. L'hétérosexualité continue de représenter l'unique cadre social de sexualité autorisé pour les professionnel·le·s. Qu'en est-il du côté des jeunes filles?

\section{DU CÔTÉ DES JEUNES FILLES :}

\section{L'APPRENTISSAGE DE L'HÉTÉROSEXUALITÉ}

"En rester à la mixité, c’est-à-dire au simple mélange, ne suffit pas. Il faut avoir le souci de réaliser un mélange à caractère éducatif ${ }^{38}$. " Ainsi s'exprime le 
docteur Le Moal en 1967. À l'instar des recherches qui ont pu être menées en sociologie sur la mixité scolaire ${ }^{39}$, il s'agit ici d'analyser la façon dont l'arrivée des éducateurs, au-delà d'une gestion des rapports de sexe, engage bien une politique pédagogique. En quoi la mixité est-elle jugée pertinente pour traiter de l'inadaptation féminine, à l'heure où les discours sur la sexualité évoluent au sein de la société.

\section{Le corps, un nouveau champ de bataille politique}

Là où les déviances des filles étaient l'objet de répression et de correction, il s'agirait à partir de la fin des années soixante de mettre le corps à l'honneur et de parler de sexualitéf ${ }^{4}$. Il est vrai qu'au cours de la seconde moitié du $\mathrm{Xx}^{\mathrm{e}}$ siècle en France, les conduites sexuelles et le regard porté sur elles ont très nettement évolué. L'accès facilité aux études, ainsi que la participation des femmes aux luttes politiques favorisent l'émergence d'une deuxième vague féministe au tournant des années soixante-dix avec la constitution du Mouvement de Libération des Femmes, visant à dénoncer le système patriarcal ${ }^{41}$. La démocratisation de la pilule en 1967 puis la dépénalisation de l'avortement contribue à libérer les femmes des contraintes qui pesaient sur leur corps et leur sexualité ${ }^{42}$. La critique de l'oppression s'étend alors à la sphère du travail, du mariage, jusqu'à la sphère domestique, dont le slogan : "le personnel est politique " est le plus emblématique. Le déclin du mariage institutionnel et les luttes des mouvements homosexuels contribuent eux aussi à élargir le champ des sexualités possibles.

De fait, l'éducation à la sexualité change sensiblement d'orientation. D'entreprise morale visant à détourner les jeunes filles des mauvaises rencontres et à favoriser la reconnaissance d'hommes à la bonne moralité, les séances intègrent peu à peu des leçons d'anatomie pour mieux comprendre le fonctionnement de son corps et les différents moyens de contraception existants. Il devient davantage question d'épanouissement et de désir : « le but de l'éducateur ou de l'éducatrice, est que l'adolescente vive bien sa sexualitét ${ }^{43}$ ». Au "desserrement » des contraintes pesant sur les éducatrices répond une volonté de remettre en cause celles imposées aux jeunes filles. Et comme pour les éducatrices, cet accès à la sexualité des jeunes filles est pensé en lien avec l'ouverture des institutions, et la volonté de se rapprocher des évolutions en cours dans la société.

\author{
39. Zaidman Claude, "La \\ mixité, objet d'étude scienti- \\ fique ou enjeu politique? ", \\ in Cahiers du genre, \\ $n^{\circ} 42 / 2007$. \\ 40. Blanchard Véronique, \\ De Brécourt à Eva. Corps \\ de jeunes filles et action \\ éducative de 1945 à nos \\ jours, Mémoire de maîtrise \\ de sciences et techniques, \\ interventions et sciences \\ sociales, mention PJJ, \\ sous la dir. de Yvorel \\ Jean-Jacques, université \\ de Versailles-Saint-Quentin \\ en-Yvelines, 1997. \\ 41. Pour aborder l'histoire \\ de cette deuxième vague \\ féministe, voit notamment \\ PIcQ Françoise, Libération \\ des femmes. Les années- \\ mouvement, Seuil, 1993. \\ 42. Voir Mossuz-Lavau Janine, \\ Les lois de l'amour. Les \\ politiques de la sexualité en \\ France (1950-2002), Paris, \\ Payot, 2002 (1991). \\ 43. Algan, La mixité du \\ groupe..., op. cit.
}


44. Voir à ce sujet : CASTARosaz Fabienne, Histoire du flirt, Grasset, 2000.

45. Voir Bozon Michel,

« Jeunesse et sexualité (1950-2000). De la retenue à la responsabilité de soi », in BANTIGNY Ludivine et JABLONKA Ivan (sous la dir.), Jeunesse oblige. Histoire des jeunes en France. $X I X^{e}-X X I^{e}$ siècle. Paris, PUF, 2009

46. Voir BLANCHARD Véronique, Revenin Régis,

YvorEL Jean-Jacques (sous la dir.), Les jeunes et la sexualité (Initiations, interdits, identités) (xix $x \times l^{e}$ siècle), Paris, Autrement, 2010.

47. Algan Andrée, «Étude comparative de la délinquance juvénile des garçons et des filles », in Les Annales de Vaucresson, $\mathrm{n}^{\circ}$ 5, 1967, CNAM p. 209

48. Algan Andrée, Nery Monique, « L'image de soi chez l'adolescente délinquante (Étude bibliographique) ", in Les Annales de Vaucresson, $n^{\circ} 6,1968$ p. 172, CNAM, P05.
Il faut dire que depuis l'ouverture des établissements scolaires à la mixité et la généralisation de l'accès à l'enseignement supérieur, la jeunesse a vu se multiplier les lieux de socialisation entre filles et garçons. Les surboums, les sorties ciné, les bals sont autant d'occasions de flirter ${ }^{44}$. À l'intérieur des institutions comme Brécourt ou Bourges, les rencontres avec des garçons deviennent possibles, lors de camps de vacances, ou de loisirs. C'est justement dans cet encadrement d'un désir hétérosexuel que la présence des éducateurs va jouer un rôle éducatif.

\section{L'éducateur, ce $(\mathrm{H})$ éros!}

Plus la sexualité juvénile gagne en autonomie, plus les inquiétudes des adultes augmentent ${ }^{45}$. Et ces craintes d'un dérèglement moral ${ }^{46}$ sont d'autant plus fortes lorsqu'il s'agit des filles, et de jeunes filles dites inadaptées ou délinquantes. En ce début des années soixante-dix, leur prise en charge par la justice continue d'être considérée comme spécifique. On parle de problématiques typiquement féminines, bien que le champ lexical ne soit plus tout à fait le même. En effet, aux discours médical et moral succède un discours plus psychologique. Aux "déviances sexuelles » sont préférées les notions de "troubles affectifs". Les jeunes filles seraient notamment " au moins aussi sensibles, sinon plus, aux tensions familiales ${ }^{47}$ ". Les recherches d'Andrée Algan intègrent aussi l'impact des facteurs sociaux sur l'estime de soi :

Le refus de son sexe propre, tout comme 《les préférences pour le rôle du sexe opposé, sont plus fréquents chez les femmes que chez les hommes, quel que soit leur âge ${ }^{48}$.» 
De coupables, les jeunes filles apparaissent d'avantage comme des êtres en souffrance psychologique ${ }^{49}$, dont les symptômes d'inadaptation se logeraient dans des troubles de l'affectivité et d'autodépréciation de leur sexe. Or, l'un des leviers de l'action éducative porte justement sur ces liens affectifs développés avec les éducateurs. Les participant·e's aux journées de Vaucresson notent qu' «à l'entrée de l'institution, les adolescentes iraient plus volontiers vers l'éducateur homme ${ }^{50}$ ". En 1972 également, les éducateurs hommes affirment qu'ils seraient davantage sollicités que leurs collègues féminines. Or, cette attirance venant de filles perturbées, seraient d'ordre érotique :

La relation de l'adolescente à l'adulte et à l'éducateur homme, plus particu《lièrement, est souvent décrite comme revendicatrice et duplice; l'entretien individuel est très généralement diffusé dans le groupe, le rapport qui en est fait tend à érotiser fréquemment la situation ${ }^{51}$. "

Andrée Algan, à son tour, en 1977 analyse le fait que « l'éducateur est celui avec lequel on ne fera pas l'amour, en dépit de l'érotisation possible de la rela$\operatorname{tion}^{52}$ ». On voit bien ici poindre l'influence des références psychanalytiques, qui se retrouvent également régulièrement invoquées dans les mémoires des élèves en formation. Selon ces grilles d'analyse, l'éducateur vient symboliser le père, et en rejouant le complexe d'CEdipe, permet l'expression d'un désir, inconscient, envers le sexe opposé. Tout comme dans les institutions de rééducation pour garçons, dans lesquelles la femme vient symboliser cet amour impossible. Cette érotisation de la relation avec l'adulte masculin n'est plus considérée comme honteuse, ou culpabilisante, mais au contraire, représente une étape nécessaire dans la construction identitaire de la jeune fille. L'éducateur est celui qui permet la réparation des troubles, et celui qui va symboliser le désir hétérosexuel : « la fille doit changer d'objet d'amour pour devenir femme, de l'attachement à sa mère, elle doit passer à l'attachement à un homme (son père d'abord, un autre ensuite), tout en continuant à s'identifier à la mère ${ }^{53}$ ». L'assurance par l'institution que ces hommes aient une sexualité claire, stable et un tant soit peu surveillée, permet alors à ce que cette attirance puisse se réaliser sans risques.

49. Voir à ce sujet la thèse de Sallee Nicolas, Des éducateurs placés sous main de justice : les éducateurs de la Protection judiciaire de la jeunesse entre droit pénal et savoirs sur l'homme, sous la direction de François Vatin, université Paris 10, 2012.

50. Rapport envoyé au ministère de la Justice, par le Centre de formation et de recherche de l'Éducation surveillée de Vaucresson, Journées des 6 et 7 mai 1971 , p. 8, Archives nationales de Pierrefitte, cote 20060575/002.

51. Ibid.

52. Algan, La mixité du groupe, op. cit., p. 51.

53. Rapport envoyé au ministère de la Justice, ibid., p. 6 . 
54. Rapport envoyé au ministère de la Justice, 1971, op. cit., p. 2

55. Entendu comme «Système asymétrique et binaire, de genre, qui tolère deux et seulement deux sexes, où le genre concorde parfaitement avec le sexe (au genre masculin le sexe mâle, au genre féminin le sexe femelle) et où l'hétérosexualité (reproductive) est obligatoire, en tout cas désirable et convenable », BUTLER Judith, Trouble dans le genre. Le féminisme et la subversion de l'identité, Paris, La Découverte, 2005,

$$
\text { p. } 24 .
$$

56. Blanchard, Revenin,

« Justice des mineurs, travail social et sexualité juvénile...», op. cit.

57. Rapport annuel de I'IPES de Brécourt de 1967, Madame Beaulu, Archives nationales de Pierrefitte, cote 19910298/65.

\section{Éviter les troubles sexuels}

En dépit de ces évolutions, la sexualité des filles constitue toujours un objet d'inquiétude, d'observation et de surveillance importante sur lequel il est nécessaire d'opérer une action éducative : «Les problèmes de la vie sexuelle apparaissent comme fondamentaux pour la rééducation des adolescentes : les conflits sexuels et affectifs seraient pour [les éducatrices] au premier plan et devraient être résolus avant de pouvoir entreprendre une action éducative positive, scolaire ou professionnelle par exemple ${ }^{54}$ ", et côté Vaucresson, Andrée Algan cite encore, dans son étude de 1974, l'influence importante des troubles hormonaux ou du développement de la puberté dans l'accroissement des comportements délictueux.

Suivant ces considérations cliniques, la présence masculine des éducateurs est perçue comme un moyen thérapeutique permettant de corriger les troubles de genre et ceux d'ordre sexuel des jeunes filles. En effet, cette fréquentation de l'autre sexe permettrait à l'adolescente de se familiariser avec les hommes, de se " réconcilier » avec eux, et d'investir de manière affective et sans risques, cette relation.

Accompagner les jeunes filles dans leur familiarisation avec l'altérité sexuelle et la sexualité permet à l'institution d'opérer un contrôle moins direct mais néanmoins efficace sur les formes de désirs jugées légitimes ou illégitimes. En effet, l'usage qui est fait de la mixité tend à promouvoir une sexualité hétéronormative ${ }^{55}$. Les tableaux portant sur les motifs de placement en internat que j'ai pu consulter confirment l'analyse établie sur la période précédente par Véronique Blanchard et Régis Révenin ${ }^{56}$ : l'homosexualité féminine - contrairement à celle des garçons -, reste taboue, cachée, même dans les dossiers éducatifs où sont dépeintes les déviances des jeunes accueillies. Lorsqu'elle est abordée, comme à Brécourt, elle est présentée comme une maladie dont il faudrait se prémunir : « Il y a un risque de contamination réelle. Tout particulièrement, les homosexuelles détectées en C.O. ne devraient jamais être envoyées en internat ${ }^{57}$. " Cette idée d'un environnement propice à l'homosexualité réapparaitt au détour des discussions sur la mixité : l'arrivée des éducateurs ou plus tard de garçons dans l'institution aurait permis de mettre un terme à ces attirances latentes : 


\footnotetext{
- Finalement, l'éducateur qui avait été nommé, je pense qu'il a sorti

《l'institution (mais c'était un peu le cas à Brécourt) d'une homosexualité patente... qui était quand même...

- Homosexualité des jeunes filles?

- Oui des jeunes filles, mais même des personnels éducatifs je pense... même si c'était pas une homosexualité réellement ${ }^{58} \ldots$ »
}

Et c'est encore les mêmes constats qui sont présentés en 1977 dans l'étude présentée par Andrée Algan : «Le travail en institution mixte perdrait sa teinture homosexuelle, néfaste pour l'équipe tout autant que pour les jeunes ${ }^{59}$."

Ainsi, aux méthodes éducatives visant à sanctionner les transgressions du genre et les déviances sexuelles, basées sur la privation, l'empêchement, le redressement des corps succèdent des méthodes disciplinaires reposant sur la production d'émotions et d'affects positifs. La sexualité devient davantage un objet à encadrer qu’à empêcher, et la présence des éducateurs, un outil éducatif susceptible de participer au processus de réadaptation des jeunes filles dites " inadaptées ». Nous serions là davantage dans ce que Michel Bozon, en reprenant le langage de Michel Foucault ${ }^{60}$, décrit comme un passage d'une sexualité construite par des contrôles et des disciplines externes à une sexualité reposant sur des disciplines internes aux individus.

\section{SeXUAlité, GENRE ET ORDRE FAMILIAL :}

\section{UN ENJEU DE CLASSEMENT SOCIAL}

À partir des années soixante, la volonté d'ouvrir les institutions sur la société s'accompagne d'une transformation des pratiques en direction des familles. L’ordonnance de 1958 sur l'assistance éducative permet de développer les mesures dites de " milieu ouvert " et demande désormais de recueillir l'adhésion des parents lors des placements. Ces mesures témoignent de l'évolution du regard porté sur des familles considérées jusqu'ici comme essentiellement mauvaises, responsables de l'inadaptation juvénile, et à ce titre, maintenues à distance des institutions ${ }^{61}$. À l'intérieur des internats de rééducation pour filles, davantage de visites sont accordées, et dans les rapports annuels apparaissent des accompagnements de la part des éducateurs au domicile familial des adolescentes. Les normes sexuelles produites par la mixité, du côté des

\author{
58. Entretien de Annie \\ Voiret, ancienne éduca- \\ trice d'abord stagiaire à \\ Brécourt, puis titulaire sur le \\ foyer de Corenc, au début \\ des années soixante-dix. \\ Entretien réalisé à son \\ domicile, le 08/06/2015 à \\ Marseille. \\ 59. Algan, La mixité du \\ groupe, op. cit., p. 10. \\ 60. Bozon Michel, op. cit. \\ 61. Yvorel Jean-Jacques, \\ «Placement et travail avec \\ les familles à l'Éducation \\ surveillée de 1945 aux \\ années soixante-dix », in \\ Revue d'histoire de l'enfance \\ « irrégulière », n 19, 2017, \\ p. 169-184.
}


62. Voir par exemple Docteur LE MOAL, "Conférence Mixité et Coéducation », op. cit.

63. Rapport envoyé au ministère de la Justice, op. cit.

64. Algan, La mixité du groupe..., op. cit. 65. Ibid. professionnel-le·s comme des jeunes filles doivent être étudiées à l'aune de ces évolutions. En effet, bien que comprises dans le cadre d'institutions fermées, elles se construisent en relation constante avec le monde extérieur, et en particulier en réaction aux troubles observés dans les familles.

\section{Des rôles éducatifs complémentaires}

Si la mixité professionnelle est relativement bien accueillie et encouragée par les personnels éducatifs, la manière dont elle doit se mettre en place est davantage discutée. À la fin des années soixante, certains écrits témoignent de la crainte d'une uniformisation des sexes ${ }^{62}$. Il faudrait veiller à maintenir une stricte différenciation. Ainsi, les journées d'étude sur la rééducation des filles en 1971 concluent : " Les rôles respectifs des éducateurs des deux sexes sont en tout cas complémentaires ${ }^{63}$. » En 1977 encore, certains professionnels affirment que la mixité doit " permet[tre] de découvrir, à travers les éducateurs et les éducatrices, des modèles masculins et féminins ", c'est pourquoi "des tâches spécifiques doivent être attribuées aux uns et aux autres afin de ne pas gêner la prise d'identité sexuelle chez les adolescents ${ }^{64}{ }^{\prime}$. Certes, la place grandissante des femmes au sein de l'Éducation surveillée et l'influence des mouvements féministes ont quelque peu interrogé les normes de genre attendues des éducatrices. Après la figure de femmes dévouées, peu visibles et peu reconnues au sein de l'administration, celles-ci souhaitent être considérées comme des professionnelles à part entière, au même titre que leurs collègues masculins. Cette évolution rencontre parfois des résistances et des discussions s'engagent sur la répartition des tâches. Mais ce qui ressort globalement des journées d'étude correspond à une revendication d'égalité, sans attenter à la différence des sexes. En effet, derrière l'universalisme des compétences éducatives, l'indifférenciation des postes, le sexe des professionnellle·s continue d'être considéré comme déterminant dans la relation établie avec les jeunes filles, les attitudes et postures éducatives : « les éducateurs assumeraient mieux les difficultés de la rééducation, en particulier les échecs ", quand "les éducatrices ressentiraient plus intensément les échecs apparents, les réussites momentanées, en un mot "colleraient" davantage à l'évolution d'un cas dans ses manifestations immédiates ${ }^{65}$ ". Cet « impact affectif profond " propre aux éducatrices relève bien ici d'une domination symbolique entre un féminin associé à l'émotionnel et un masculin qui se trouverait, lui, du côté d'un rationnel plus valorisant. Au Centre de recherche 
et de formation de Vaucresson, le courant de psychologie différentielle auquel Andrée Algan appartient contribue à diffuser l'idée de caractères psychologiques proprement masculins et d'autres rattachés à une nature féminine. Et cette différence est renforcée par l'organisation matérielle du travail : les hommes sont majoritaires dans les activités extérieures sportives, de loisirs ou dans les structures hors les murs de l'institution, comme le milieu ouvert. Concernant l'accès aux postes de pouvoir, à Bourges, comme à Brécourt, ils possèdent plus de responsabilités, soit selon une logique horizontale en cumulant des fonctions, soit selon une logique verticale, en accédant majoritairement aux fonctions des cadres. Au-delà de cette organisation matérielle, les discours portés par les directions tendent aussi à produire une différenciation sexuée. À Bourges, il apparaît dans les rapports annuels que l'embauche d'hommes permettrait de faire de la médiation entre les jeunes filles et les garçons de l'extérieur : "D'autre part, nos filles sont très rapidement en contact avec des garçons, ceci amène un conflit entre les exigences éducatives et l'intervention d'un homme peut être efficace à ce niveau ${ }^{66}$. " À Brécourt, Dominique Riehl est en proie à des conflits importants avec son équipe. Elle espère alors que l'arrivée des éducateurs permettra de régler les conflits. Les hommes continuent d'être pensés comme garants d'une certaine autorité. Par le biais de la mixité, l'organisation institutionnelle reproduit donc une différenciation et une séparation entre les sexes, basée sur la domination masculine. Les professionnel-le·s restent des individus sexués, et si les normes de genre évoluent, elles continuent de caractériser la manière dont éducatrices et éducateurs doivent se comporter, et dont le travail doit être organisé. Cette division sexuée des rôles et des tâches est d'autant plus difficile à remettre en cause qu'elle est justifiée d'un point de vue éducatif, à partir des concepts de couples éducatifs et les références faites au modèle familial.

\section{La promotion d'un modèle familialiste}

Le modèle de féminité que les éducatrices se doivent d'incarner, du temps de la non-mixité, est souvent comparé au rôle maternel. Le dévouement des religieuses ou des laïques ne serait finalement qu'une prolongation, une extension du « devoir naturel » des femmes. Avec l'arrivée des éducateurs et la défense de la mixité des équipes, cette analogie se renouvelle, en empruntant cette fois-ci au vocable de la conjugalité. Plutôt que de vrais ménages, les différents acteurs sociaux font savoir leur préférence pour la forme fictive des couples éducatifs. Inscrite dans
66. Rapport annuel de Bourges, "Section IV. Internat, remarques générales sur l'organisation pédagogique », 1971 , p. 5, Archives nationales de Pierrefitte, cote 19910298/81. 
67. Jovinot Étienne (rapporteur) « Le profil de l'éducateur spécialisé en 1968, et perspectives d'avenir ", Compte-rendu du colloque organisé par le Comité d'entente des écoles et centres de formation d'éducateur spécialisé au Centre de recherche et de formation de l'Éducation surveillée de Vaucresson, 19-23 février 1968, in « La formation de l'éducateur spécialisé de jeunes inadaptés", in Sauvegarde de l'Enfance, no 12 , novembredécembre 1968. p. 539 CEDIAS-Musée social.

68. « ENFPES de Toulouse Mémoires rédigés. Promotion 1971-1973 », SImon, "La mixité des équipes éducatives en internat de garçon ", p. 15, Archives nationales de Pierrefitte, cote 20110313/12

69. Algan, La mixité du groupe..., op. cit., p. 40.

70. Docteur MATHIS, "L'enfant privé de sa famille », in Sauvegarde de l'Enfance, p. 209, 1969, CEDIAS-Musée social. un cadre institutionnel, la conjugalité correspond davantage à une mise en scène, à une représentation, jugée nécessaire dans le processus de construction identitaire des adolescentes. Ainsi, la session de formation organisée à Vaucresson en 1968 sur « le profil de l'éducateur » estime que la présence d'un couple auprès des jeunes, est " maintenant nécessaire à l'éducation des garçons et des filles de tout âge ${ }^{67}$ ». Cet intérêt éducatif apparaît également dans les écrits d'élèves en formation : " la mixité de l'équipe éducative fournira aussi, aux jeunes placés en internat, une image de couple ${ }^{68}$ ». De même, le rapport d'Andrée Algan en 1977 conclut que, parmi les couples éducatifs, certains pourront incarner « davantage des références parentales ${ }^{69} »$. Enfin, les critiques présentes dans les rapports des deux directions, de Brécourt et de Bourges, sur l'immaturité des éducatrices sousentendent que l'écart d'âge de professionnel·le·s plus mûr·es, ou l'embauche de personnels mariée's permettraient davantage la représentation de figures parentales. À Brécourt, il est d'ailleurs fait mention à deux reprises de la qualité « sainement maternelle » d'une éducatrice. Celle-ci est mariée et il est précisé qu' elle est « en espoir de maternité ». L'expérience ou les qualités parentales sont considérées comme des gages de qualités éducatives.

La présence du couple est aussi parfois perçue comme salutaire d'un point de vue psychique. Ainsi, le docteur Mathis qui s'exprime en 1969 dans les colonnes de la Sauvegarde de l'Enfance considère à propos de l'éducation en internat que :

Cette image d'identification [celle de l'éducatrice] reste cependant insuffi《sante. Il y manque l'image masculine, paternelle, de ce fait, il n'y a pas non plus d'image de couple. La présence d'hommes jouant un rôle assez important ou la direction du groupe par un couple pourrait y suppléer ${ }^{70}$. »

Nous retrouvons ici les influences de la psychologie et plus particulièrement de la psychanalyse. De nombreuses publications scientifiques insistent sur les troubles occasionnés par l'absence du père. L'arrivée d'hommes dans l'équipe pourrait représenter un intérêt thérapeutique pour les jeunes filles, en venant d'abord combler un manque et faire tiers dans la relation entre les adolescentes et des éducatrices dépeintes comme trop investies affectivement, ou en conflit. La mise en place de couples éducatifs permettrait ainsi, en représentant un ordre familial traditionnel de ré-affirmer la différence des sexes : aux éducatrices le rôle maternel, aux éducateurs, l'autorité paternelle. Ceci, au moment même où les discours se multiplient sur les carences affectives provoquées par l'absence ou la 
démission des pères ${ }^{71}$. C'est pourquoi il convient de comprendre cette mise en place et cette défense des couples éducatifs en écho aux discours produits par les institutions sur la famille. La mixité ne viendrait-elle pas signifier la remise à l'endroit des défaillances observées en termes de genre dans l'ordre familial ?

Andrée Algan travaille en 1969 auprès de l'équipe éducative du tribunal pour enfants de Verdun. Elle est la rapporteuse de leur étude sur les adolescentes inadaptées et délinquantes, et à ce titre écrit : " la famille est considérée comme incapable de remplir son rôle donc mauvaise, et l'éducateur ou le centre vient se substituer à cette famille déficiente ${ }^{72} "$. Sans aller jusqu’à parler de remplacer les parents, les participant·e·s aux journées sur les foyers en 1977 partagent l'idée que l'éducateur vient apporter un " re-père » identificatoire :

Il est évident que le rôle de l'éducateur, s'il comporte une part d'engagement 《affectif, est autre que de vouloir se substituer à la famille, si celui-ci accepte, à un moment donné d'être une figure identificatoire, d'être un repère (un re-mère) c'est dans une optique thérapeutique qui met en jeu des phénomènes de transfert et de contre-transfert difficiles à saisir et à contrôler en dehors d'un solide travail d'équipe ou d'une supervision ${ }^{73}$.»

De même, la direction de Brécourt propose dans un de ses rapports annuels de faire fonctionner les pavillons ${ }^{74}$ à l'image d'un "foyer ", avec un couple responsable à sa tête. Ce souhait semble se réaliser puisque les tableaux d'effectifs font apparaître des binômes éducatifs quasi systématiquement. L'encadrement des jeunes filles par un couple marié favorise la porosité entre identité professionnelle et représentation d'un modèle parental. Enfin, ces structures, type "foyers d'action éducative " qui petit à petit prennent le pas sur les grosses institutions de type internat, ne cherchent-elles pas implicitement la référence à une ambiance plus "familiale "? Ces expressions sont en tout cas toujours d'actualité et l'on peut encore en trouver mention dans de nombreux projets institutionnels. Ainsi, nous serions bien en présence d'un processus de familialisation ${ }^{75}$ des rapports entre les professionnel-le's et les jeunes filles, notamment lorsqu'il y a présence de couples éducatifs. Le rôle des équipes est souvent associé à des références parentales, et cette représentation de figures maternelles et paternelles est utilisée comme un outil thérapeutique. Cette familialisation constituerait in fine un levier éducatif utile pour répondre aux troubles identitaires des jeunes filles, provoqués entre autres par des modèles parentaux jugés problématiques
71. Comme en témoigne par exemple l'article du Docteur HEURTEVEnt Jean, "Contribution à l'étude du traitement et de la rééducation des adolescentes déséquilibrées caractérielles très difficiles ", in Rééducation, $n^{\circ} 222,25$ e Année, juin 1970, p. 3-4.

72. Algan, «Étude comparative de la délinquance juvénile... », op. cit., p. 173.

73. " Compte-rendu de la session sur les foyers, 25-26 mai 1977 », p. 5, Archives nationales de Pierrefitte, cote 19910333/3 F 1348.

74. Les pavillons désignent les groupes sur lesquels sont accueillies les jeunes filles.

75. J'emprunte ici le terme de " familialisation » des relations entre usagers et professionnels aux auteurs du rapport « Le genre de l'autonomie »: BEssin Marc (dir.) et al., Le genre de l'autonomie. Enquêtes sur la sexuation des interventions sociales, rapport de recherche pour la MIRE/ DARES, IRIS-CSPRP, avril 2009 . 
76. WaCQUANT LoÏc, LeS Prisons de la misère, Éditions Raisons d'Agir,

1999.

77. SkEgGs Beverley, Des femmes respectables.

Classe et genre en milieu populaire, Marseille, Agone, 2015.

78. Algan, « Étude comparative de la délinquance juvénile... », op. cit., p. 164. en termes de rapports et d'identité de genre. De la même manière, le modèle de conjugalité hétérosexuelle, même fictif, devient plus facile à envisager que du temps de la non-mixité lorsque les éducatrices, célibataires, devaient apprendre aux jeunes filles à être de bonnes épouses.

\section{La sexualité, un enjeu de différenciation sociale?}

Les différents tableaux statistiques de l'époque sur l'origine sociale des jeunes filles mettent en évidence une sur-représentation des classes populaires. De nombreux sociologues - Loïc Wacquant entre autres ${ }^{76}$ - ont révélé que cette situation, loin de signifier que les pauvres seraient naturellement plus dangereux et plus délinquants, résulte en grande partie d'un processus de criminalisation de la misère; les pauvres, seraient d'abord davantage l'objet de mesures coercitives et répressives de la part de l'État. Le traitement et la définition de la délinquance ont donc à voir avec le traitement de la question sociale.

L'évolution des orientations éducatives vise à prendre davantage en compte la famille, mais les relations entre celle-ci et les éducateurs et les éducatrices n’en sont pas moins investies par des rapports de pouvoir, dus en partie à la structure des rapports socio-économiques, mais du fait également que l'Éducation surveillée exerce un pouvoir judiciaire et un pouvoir symbolique sur les familles. En effet, au-delà des décisions de placement, par ses discours et ses pratiques institutionnelles elle produit des valeurs morales et des modèles culturels. La sociologue Beverley Skeggs a brillamment démontré comment s'exercent les mécanismes de domination culturelle de la classe bourgeoise sur les filles des milieux populaires, à partir des normes de féminité et de sexualitét7. De la même manière les discours sur la mixité montrent que la sexualité constitue un vecteur de classement social.

C'est bien à partir de transgressions de genre et de sexualité que les familles sont jugées problématiques. Ainsi, après avoir noté que leur vie conjugale est parfois « caractérisée par une domination excessive de la femme et par une démission du mari [et que] la structure de certaines familles se rapproche du type matriarcal ${ }^{78}$ ", Andrée Algan rapporte :

À l'opposé de l'image maternelle, apparaît pour le père une image falote; 《le père est réduit à l'état de fantôme; il n'existe nulle part [...] L'image maternelle que reçoivent les adolescentes est celle d'une femme dominant son 
mari, mais son autorité sur ses enfants est jugée par l'entourage social comme responsable de la situation du foyer. L'image paternelle est celle d'un homme irresponsable, démissionnaire, castré de son rôle social et par suite humiliante pour l'adolescente ${ }^{79}$."

Cette absence des pères est jugée néfaste pour Andrée Algan qui affirme en 1967 que :

pour la fille, l'image paternelle étant chargée de lui fournir un support, un 《soutien, son absence a de grave répercussions : dans certains cas, l'adolescente ne peut percevoir les fonctions et les rôles normaux de l'homme et de la femme dans la famille : elle cherche alors une solution à ses incertitudes dans la pratique sexuelle; parfois aussi, son inconduite sexuelle peut s'expliquer par des formations réactionnelles : haine du père ayant abandonné le foyer, ou du beau-père, et auto-punition due à de profonds sentiments de culpabilités ${ }^{80}$.

Dans ce premier modèle de pensée, les femmes des milieux populaires sont associées à l'excès, au débordement, aux transgressions des lois dites « naturelles ${ }^{81}$ ".

Face à cela, l'encadrement institutionnel de la sexualité vise à apprendre aux jeunes filles à être respectables. Il s'agit d'apprendre à se maîtriser, et à savoir tenir son rôle de femme, voire d'épouse. À Brécourt, la direction considère que les mariages contractés par les jeunes filles représentent un critère de réadaptation réussie.

Mais un autre registre discursif est présent dans les écrits, qui disqualifie tout autant les familles. Il consiste à associer les milieux populaires à la défense d'un ordre conservateur, traditionnel, archaïque, incapables de prendre en compte les évolutions en cours, contrairement aux classes moyennes représentées comme plus progressistes. Ainsi, le docteur le Moal, dans sa conférence sur «Mixité et coéducation " constate que " les milieux populaires sont plus attachés dans l'ensemble aux images traditionnelles, ici aux images-types que l'on se fait de l'homme et de la femme ${ }^{82}$ ». Cette idée apparaît aussi derrière la question posée par les organisateurs des journées de Vaucresson :

- En milieu ouvrier, une image assez traditionnelle de la femme consacrée $\ll_{\text {uniquement au foyer semble encore fréquente. Pensez-vous que les femmes }}$
79. Algan, «Étude comparative de la délinquance juvénile... », op. cit., p. 165.

80. Ibid.

81. Il est intéressant de noter que ce sont les mêmes constats que formule Hélène LE DANTEC-LOWRY quand elle analyse les discours produits sur les familles noires aux États-Unis : le rapport de Daniel $P$. Moynihan pour l'administration Johnson reprend quasiment les mêmes jugements de valeur pour prouver que ces familles sont dysfonctionelles car matriarcales. Voir LE DANTEC-LOWRY Hélène, De l'esclave au Président: discours sur les familles noires aux États-Unis, Paris, CNRS Editions, 2010.

82. Docteur Le Moal, "Conférence Mixité et Coéducation... ", op. cit., p. 9. 
83. « Journée d'études sur la rééducation des filles inadaptées », Vaucresson, 22-25 avril 1970, p. 2, Archives nationales de Pierrefitte, cote 20060575/002.

84. Rapport envoyé au ministère de la Justice, op. cit.

85. Algan Andrée, La mixité du groupe, op. cit., p. 24. de ce milieu puissent avoir, actuellement, d'autres formes d'action ou d'autres modes de réalisation personnelle? - Dans les classes moyennes, des images de femmes plus indépendantes apparaissent; dans ce cas, quelles sont les réactions des hommes de ce milieu, et ces réactions sont-elles liées à la situation professionnelle qu'ils occupent ${ }^{83}$ ? »

L'année suivante, c'est en des termes culturels que réapparaît cette idée :

Par ailleurs, les cultures traditionnelles, valorisant le statut masculin, rendent $\ll$ plus malaisé pour elle que pour le garçon une identification à son propre rôle sexuel ${ }^{84}$.»

Qu'elles transgressent les normes de féminité ou qu'elles incarnent des modèles trop traditionnels, les mères ne semblent jamais correspondre aux attentes sociales. Dans ce contexte, la mixité, synonyme d'avancée sociale par rapport à l'entre-soi qui prévalait jusqu'alors, représente un progrès également en termes de modernité sexuelle. En effet, toujours selon une grille culturaliste, les professionnel-le·s interrogé·e·s par Andrée Algan considèrent que «les familles nord-africaines tolèrent en général très difficilement une formule mixte pour leurs filles ${ }^{85}$ ". Permettre cette fréquentation de l'autre sexe, quand bien même il s'agit d'adultes, semble être le signe d'un progrès social, face aux mentalités fermées de certains milieux populaires et de familles immigrées. De même, l'introduction de la contraception, voire la défense de l'avortement sont parfois défendus par les professionnel-le's comme des moyens d'émancipation des filles de leurs familles. Alors que les motifs et les formes de placement des jeunes filles continuent de mettre en évidence la pénalisation des transgressions des normes de genre et de sexualité, certains discours des professionnelle·s et des chercheur. euse's de l'Éducation surveillée tendent à représenter les institutions comme les lieux d'apprentissage de l'épanouissement et de l'autonomie sexuelle.

\section{ConClusion}

Si la présence des deux sexes au sein des équipes professionnelles à la PJJ ne fait plus débat aujourd'hui, l'étude de cette arrivée des éducateurs dans les internats de rééducation pour filles nous montre comment, loin d'être fortuite, sa mise en place répond à de multiples enjeux, et comment, un peu forcée par 
les événements, elle se trouve rapidement justifiée d'un point de vue éducatif, comme une nouvelle façon de répondre aux problèmes d'inadaptation féminine.

Dans un contexte de bouleversements importants tant dans la société qu'au sein de l'Éducation surveillée, avec l'arrivée d'une jeune génération de professionnel·le·s, l'apparition de nouvelles revendications portant notamment sur la question de la liberté sexuelle, ou encore la multiplication des lieux de rencontre entre hommes et femmes, la mixité professionnelle apparaît comme une nécessité et un signe d'ouverture des institutions.

Du temps de la non-mixité, l'analogie entre le traitement des éducatrices et des jeunes filles est évident; isolées, surveillées, elles sont assignées les unes comme les autres à des normes de féminité très contraignantes. Selon cet idéal de vertu féminine, la possibilité de désirs sexuels à l'intérieur de l'institution est niée, empêchée, impensable même. C'est le célibat pour les éducatrices, l'attente du mariage pour les adolescentes. En cette fin des années soixante, les jeunes professionnelles sont comme les adolescentes, jugées immatures, et en proie à une sensiblerie et des troubles affectifs. La présence des éducateurs représente alors une certaine mise en ordre, mais aussi bouscule la place de la sexualité, aussi bien pour les éducatrices, qui vont pouvoir s'émanciper du célibat obligatoire, que pour les jeunes filles. Au modèle de l'imitation succède celui de l'identification de la différence sexuée. La manière d'agencer les sexes, en s'appuyant sur les modèles des couples éducatifs et les références parentales offrent ainsi aux jeunes filles un nouveau modèle d'hétéronormativité légitime. Loin d'être une véritable libération sexuelle, la mixité correspond davantage à un réagencement des normes, à partir de nouveaux savoirs et de techniques disciplinaires. De la répression des désirs on passe à des mécanismes plus intérieurs de contrôle de soi. L'individu est davantage considéré à partir de références psychologiques et psychanalytiques.

L'observation de la sexualité s'invite alors aussi dans la famille. Les défaillances des parents sont désormais regardées sous l'angle des rapports de genre et de sexualité. Se dessine alors un ordre social au sein duquel certains cadres de la conjugalité, produits par l'institution sont justifiés, quand d'autres, associés aux milieux populaires dont sont issues ces jeunes filles, sont disqualifiés.

La délinquance juvénile n'en finit pas d'être l'objet d'angoisses sociales. Les travaux de Stéphanie Rubi ${ }^{86}$ notamment, démontrent bien la façon dont les bandes de filles d'aujourd'hui continuent d'être traitées sous l'angle d'une
86. RuBi Stéphanie, Les "crapuleuses", ces adolescentes déviantes, Paris, PUF, 2005. 
87. C'est notamment la thèse d'auteurs tels qu'Hugues Lagrange dans son ouvrage Le déni des cultures, Paris, Seuil, 2010. transgression des normes de genre et de sexualité. En outre, l'idée que l'origine de cette délinquance serait liée à des dysfonctionnements de l'ordre sexué et sexuel des familles, n'a pas perdu de son audience. Doublée d'une approche culturaliste associant ces problématiques à certains groupes sociaux immigrés ou supposés immigrés ${ }^{87}$, ces grilles de lecture prouvent combien la sexualité et les rapports entre les genres continuent à être affaire de pouvoir et de contrôle social.

\section{Bibliographie}

"Sexe : sous la révolution, les normes ", Mouvements, vol. 20, n 2, 2002.

Artieres Philippe et Zancarini-Fournel Michelle (dir.), 1968, une histoire collective (1962-1981), Paris, La Découverte, 2008.

Berard Jean, "De la libération des enfants à la violence des pédophiles. La sexualité des mineurs dans les discours politiques des années 1970 ", Genre, sexualité \& société, $\mathrm{n}^{\circ} 11$, printemps 2014.

Bessin Marc (dir.) et al., Le genre de l'autonomie. Enquêtes sur la sexuation des interventions sociales, rapport de recherche pour la MIRE/DARES, IRISCSPRP, avril 2009.

Blanchard Véronique, De Brécourt à Eva. Corps de jeunes filles et action éducative de 1945 à nos jours, mémoire de maîtrise de sciences et techniques, interventions et sciences sociales, mention PJJ, sous la dir. de Yvorel JeanJacques, université de Versailles-Saint-Quentin en-Yvelines, 1997.

Blanchard Véronique, Revenin Régis et Yvorel Jean-Jacques (sous la dir.), Les jeunes et la sexualité (Initiations, interdits, identités (XIX'-XXI siècle), Paris, Autrement, 2010.

Blanchard Véronique et Revenin Régis, "Justice des mineurs, travail social et sexualité juvénile dans le Paris des années 1950 : une prise en charge genrée ", Les Cahiers de Framespa, [en ligne], n 7, 2011, mis en ligne le 6 avril 2011.

Blanchard Véronique, "Mauvaises filles " : portraits de la déviance féminine juvénile (1945-1958), thèse de doctorat en histoire, sous la dir. de Chauvaud Frédéric, Poitiers, 2016.

Bozon Michel, «Jeunesse et sexualité (1950-2000). De la retenue à la responsabilité de soi ", in BANTignY Ludivine et JABLONKA Ivan (sous la dir.), Jeunesse oblige. Histoire des jeunes en France. XIX'-XXI siècle, Paris, PUF, 2009. 
Butler Judith, Trouble dans le genre. Le féminisme et la subversion de lidentité, Paris, La Découverte, 2005.

Casta-Rosaz Fabienne, Histoire du firt, Grasset, 2000.

Courson J.-P. et Saboulin M., "Ménages et familles : vers de nouveaux modes de vie? ", Économie et statistiques, n ${ }^{\circ} 175,1985$.

Gardet Mathias, Peyre Vincent et Tetard Françoise (dir.), Elles ont épousé l'éducation spécialisée. Éducatrices et femmes d'éducateurs il y a cinquante ans, Conservatoire national des archives et de l'histoire de l'Éducation spécialisée, L'Harmattan, 1999.

Guilpain Geneviève, Les célibataires, des femmes singulières. Le célibat en France (XVII $-X X I^{e}$ siècle), Paris, L'Harmattan, 2012.

Kergoat Danièle, Vilbrod Alain et Guichard-Claudic Yvonne (dir.), L'inversion du genre. Quand les métiers masculins se conjuguent au féminin... et réciproquement, PUR, 2008.

Koeppel Béatrice, De la pénitence à la sexologie : essai sur le discours tenu aux jeunes filles, Le Sycomore, 1982.

Lagrange Hugues, Le déni des cultures, Paris, Seuil, 2010.

Lagrave Rose-Marie, "Une émancipation sous tutelle. Éducation et travail des femmes au Xx $x^{\mathrm{e}}$ siècle ", in Duby Georges et Perrot Michelle (dir.), Histoire des femmes en Occident, tome 5 : Le XX siècle, Paris, Plon, 1991.

Le Dantec-Lowry Hélène, De l'esclave au Président : discours sur les familles noires aux États-Unis, Paris, CNRS Éditions, 2010.

Lefaucheur Nadine, 1992, "Maternité, famille, État", in Duby Georges et Perrot Michelle (dir.), Histoire des femmes en Occident, tome 5 : Le XXe siècle, Paris, Plon, 1991.

Mossuz-Lavau Janine, Les lois de l'amour. Les politiques de la sexualité en France (1950-2002), Paris, Payot, 2002 (1991).

Perrot Michelle, Les femmes ou les silences de l'Histoire, Paris, Flammarion, 1998.

PICQ Françoise, Libération des femmes. Les années-mouvement, Seuil, 1993.

Rogers Rebecca (dir.), La mixité dans l'éducation : enjeux passés et présents, Lyon, ENS, 2004.

Rubi Stéphanie, Les «crapuleuses», ces adolescentes déviantes, Paris, PUF, 2005. 
Sallee Nicolas, Des éducateurs placés sous main de justice : les éducateurs de la Protection judiciaire de la jeunesse entre droit pénal et savoirs sur l'homme, sous la direction de François VATIN, université Paris 10, 2012.

Schweitzer Sylvie, Les femmes ont toujours travaillé. Une histoire du travail des femmes aux XIX et XX' siècles, Paris, Odile Jacob, 2002.

Skeggs Beverley, Des femmes respectables. Classe et genre en milieu populaire, Marseille, Agone, 2015.

Thomazeau Anne, "Entre éducation et enfermement : le rôle de l'éducatrice en internat de rééducation pour filles, de la Libération au début des années 1960 ", Revue d'histoire de l'enfance "irrégulière », n 7, 2005.

WacQuant Loïc, Les prisons de la misère, Éditions Raisons d'Agir, 1999.

Yvorel Jean-Jacques, "Placement et travail avec les familles à l'Éducation surveillée de 1945 aux années soixante-dix ", in Revue d'histoire de l'enfance "irrégulière ", nº 19, 2017

Zaidman Claude, "La mixité, objet d'étude scientifique ou enjeu politique?", in Cahiers du genre, $\mathrm{n}^{\circ}$ 42, 2007.

\section{SOURCES IMPRIMÉES}

Rapport annuel de l'IPES de Brécourt de 1967, Madame Beaulu, Archives nationales de Pierrefitte, cote 19910298/65.

"Bulletin du Syndicat National des Personnels de l'Éducation Surveillée ", n 46, décembre 1964, Archives de Savigny-sur-Ogre, sans cote.

Algan Andrée, "Étude comparative de la délinquance juvénile des garçons et des filles ", in Les Annales de Vaucresson, n 5, 1967, CNAM, cote P05.

Algan Andrée, Nery Monique, "L'image de soi chez l'adolescente délinquante (Etude bibliographique) ", in Les Annales de Vaucresson, n 6, 1968.

Algan Andrée, La mixité du groupe dans les institutions accueillant des jeunes inadaptés. Rapport d'enquête, Éd. Vaucresson, Centre de formation et de recherche de l'Éducation surveillée, avril 1977.

Algan Andrée, "Compte-rendu de la session sur la mixité du groupe dans les institutions accueillant des jeunes inadaptés. Vaucresson, du 16-20/1/1978 ", mars 1978, Archives de Savigny-sur-Orge, sans cote.

Pretot Renée, "Hypothèse de travail : le complexe éducatif ", in Les Annales de Vaucresson, 1973, $\mathrm{n}^{\circ} 11$. 
RieHl Dominique, "L'éducatrice d'IPES ", compte rendu d'une conférence de la directrice de l'IPES de Brécourt, session de formation des éducateurs, 1947, Archives de Savigny-sur-Orge, sans cote.

Rapport envoyé au ministère de la Justice, par le Centre de formation et de recherche de l'Éducation surveillée de Vaucresson, Journées des 6 et 7 mai 1971, Archives nationales de Pierrefitte, cote 20060575/002.

"Colloque de 1968 sur la formation de l'éducateur à Vaucresson en février 1968, organisé par le comité d'entente des écoles et centres de formation d'éducation spécialisée : session sur sélection des candidats éducateurs en 1968, rapporté par $M^{\text {lle }}$ Bauer ", in Sauvegarde de l'enfance, 12, $\mathrm{n}^{\circ} 553$, 1968, CEDIAS-Musée social.

" Journées d'études sur les structures d'action éducative destinées aux filles inadaptées Vaucresson 20-22 mars 1972 ", Archives nationales de Pierrefitte, cote 20060575/002.

Rapport envoyé au ministère de la Justice, par le Centre de formation et de recherche de l'Éducation surveillée de Vaucresson, Journées des 6 et 7 mai 1971, p. 8, Archives nationales de Pierrefitte, cote 20060575/002.

Docteur Le Moal, "Conférence Mixité et Coéducation », Centre catholique d'éducation familiale (France), 1968, Bibliothèque nationale de France, cote O-R-62300 (1967-1968,5).

«ENFPES de Toulouse. Mémoires rédigés. Promotion 1971-1973 », «SIMON, La mixité des équipes éducatives en internat de garçon ", p. 15, Archives nationales de Pierrefitte, cote 20110313/12.

Docteur Mathis, "L'enfant privé de sa famille ", in Sauvegarde de l'Enfance, p. 209, 1969, CEDIAS-Musée social.

Docteur Heurtevent Jean, "Contribution à l'étude du traitement et de la rééducation des adolescentes déséquilibrées caractérielles très difficiles ", in Rééducation, $\mathrm{n}^{\circ} 222,25^{\mathrm{e}}$ année, juin 1970.

"Compte-rendu de la session sur les foyers, 25-26 mai 1977 », p. 5. Archives nationales de Pierrefitte, cote 19910333/3 F 1348.

"Journée d'études sur la rééducation des filles inadaptées ", Vaucresson 22-25 avril 1970, p. 2. Archives nationales de Pierrefitte, cote 20060575/002. 